\title{
Éditorial: la vie d'une revue scientifique
}

Lorsque j'ai commencé la composition de ce numéro, j'ai été frappé par le fait qu'il marque la $25^{\text {ième }}$ année de publication de la revue, laquelle est donc passée de l'enfance à l'adolescence et enfin à l'âge adulte. $\mathrm{Au}$ début, je comptais m'inspirer des propos des rédacteurs en chef qui m'ont précédé, mais après réflexion, j'ai décidé de tourner résolument mon regard vers l'avenir plutôt que vers le passé.

Cet anniversaire et ce numéro marquent le début d'une série d'événements importants dans la vie de la revue. En premier lieu, mentionnons certains changements proposés et acceptés par le conseil de l'ACG dans la structure du comité de rédaction, au résultat desquels nous avons créé un nouveau poste au sein du comité de rédaction, celui de rédacteur en chef adjoint, et décidé de constituer un comité consultatif de rédaction. Le rédacteur en chef adjoint travaillera de concert avec le rédacteur en chef et les responsables de section pour améliorer l'efficacité du processus de rédaction. Dans ce numéro figure un appel de candidatures décrivant le profil souhaité pour le poste de rédacteur en chef adjoint. J'espère que ceux et celles de nos membres qui ont une expérience significative de la rédaction et dont les qualités correspondent au profil décrit n'hésiteront pas à communiquer avec moi au sujet de ce poste. J'ai demandé aux responsables des différentes sections de recommander des chercheurs œuvrant au sein d'institutions canadiennes et internationales, que j'inviterai à faire partie de notre comité consultatif de rédaction. $\mathrm{Au}$ terme de ce processus, le comité consultatif sera formé de huit chercheurs distingués (quatre du Canada et quatre de l'étranger), qui représenteront les quatre sections de la revue. Leur rôle sera de conseiller les rédacteurs et de collaborer avec les responsables de section pour procéder au choix d'évaluateurs qualifiés.

Le second changement qui aura lieu en cours d'année est l'introduction d'un processus électronique de dépôt des manuscrits. Je suis actuellement en train de tester le logiciel qui sera utilisé et, d'ici la fin de l'année, nous serons en mesure d'offrir ce service à nos auteurs. Notre objectif est d'éliminer progressivement le dépôt de manuscrits sur papier, qui disparaîtra graduellement au fur et à mesure que les auteurs se familiariseront avec le nouveau mode de dépôt électronique.

Ma première année comme rédacteur en chef se distingue surtout pour les réactions positives que j'ai reçues. J'ai apprécié tout particulièrement le travail réalisé par les responsables de section et les évaluateurs au cours de l'année, et je les remercie explicitement à la fin de ce numéro. Le seul aspect de la revue qui $\mathrm{m}^{\prime}$ inquiète, cependant, $\mathrm{c}^{\prime}$ est le nombre élevé d'auteurs qui m'ont signalé la lenteur du processus de décision concernant l'acceptation de leur manuscrit. Je suis certain que les changements décrits plus haut contribueront à rendre le processus d'évaluation généralement plus efficace, mais je tiens à rappeler à tous les lecteurs de la $R C V$ que, si on leur demande de faire le compte-rendu d'un manuscrit, il est impératif qu'ils le fassent dans les délais prescrits par les rédacteurs. En effet, s'il est vrai que nous ne pouvons maintenir le niveau de qualité établi par les rédacteurs en chef précédents sans disposer d'évaluateurs hautement qualifiés, nous ne pouvons pas non plus nous permettre de frustrer nos potentiels auteurs à cause d'un processus de décision qui s'avère d'une lenteur inacceptable.

Un troisième événement a eu lieu dont nos lecteurs ne sont peut-être pas au courant: les articles publiés par la $R C V$ depuis 2004 sont maintenant disponibles en version électronique dans les bibliothèques qui participent au Projet MUSE. Le Project MUSE est une collaboration entre les bibliothèques et les éditeurs, qui donne accès en ligne à plus de 300 revues scientifiques, publiées par 60 éditeurs, dans le domaine des sciences humaines, des arts et des sciences sociales, ce qui permet facilement de télécharger le texte intégral de tous les articles à prix très économique (http://muse.jhu.edu/about/muse/ overview.html). Pour les membres des institutions abonnées au Project MUSE, la version électronique des articles est gratuite. Grâce à cette initiative, la $R C V$ est donc disponible dans des centaines d'universités et de bibliothèques institutionnelles de tout le Canada, ainsi que dans de nombreux autres pays: Afrique du Sud, Allemagne, Argentine, Australie, Belgique, Chine, Costa Rica, Danemark, Égypte, Espagne, France, Grèce, Hong Kong, Inde, Israël, Italie, Japon, Mexique, Pays-Bas, Nouvelle Zélande, Norvège, Palestine, Pologne, Qatar, République de Corée, Roumanie, Royaume-Uni, Singapour, Suède, Suisse, Turquie et États-Unis. Grâce au Project MUSE, les articles de la $R C V$ sont maintenant lus par les chercheurs du monde entier, mais aussi par tous ceux qui s'intéressent à la gérontologie, outre les membres de l'ACG et ses abonnés institutionnels. 
Des changements sont également prévus pour ce qui est des contenus de la $R C V$. Tout au long des années 1990 et pendant les premières années de ce nouveau siècle, la recherche en gérontologie a connu un grand essor au Canada. Le Réseau canadien de recherche sur le vieillissement, le Programme de recherche sur l'autonomie des aînés, l'Étude canadienne sur le vieillissement et plus récemment les Dimensions socioéconomiques du vieillissement de la population, le projet «Hidden Costs / Invisible Contributions Major Collaborative Research Initiatives» et bientôt l'Étude longitudinale canadienne sur le vieillissement, sont chacun à sa manière le reflet de ce processus et sont représentatifs d'une série de programmes de recherche importants. De nombreux aspects de ces projets ont trouvé leur place dans les pages de la $R C V$, mais je crois qu'il reste toutefois une lacune importante à combler, celle de fournir aux chercheurs principaux de programmes de recherche particulièrement importants l'occasion de présenter ce qu'ils ont accompli. Dans ce numéro, j'ai donc décidé d'introduire une nouvelle rubrique spécialisée pour combler ce vide, et je compte à l'occasion y publier un article, en français et en anglais, entièrement consacré à un programme de recherche s'étalant sur plusieurs années qui, selon les rédacteurs, mérite d'être souligné. À ce propos, j'espère que tous liront l'article de François Béland et al. qui figure dans ce numéro, et que les lecteurs me feront connaître leur opinion sur l'opportunité de publier d'autres articles de ce type dans les prochains numéros.

Il me fait également très plaisir que la revue semble attirer de plus en plus de manuscrits provenant de sources internationales, ainsi que des articles centrés sur les processus qui sous-tendent les questions de santé fondamentales pour une population vieillissante. Je crois que ce succès témoigne du fait que le public de la $R C V$ est de plus en plus nombreux, grâce entre autres à la diffusion du Project MUSE, au fait que la revue soit maintenant cataloguée par Medline et aux ressources supplémentaires mises à notre disposition par Instituts de recherche en santé du Canada - Institut du vieillissement. Bien que nous ayons de nombreux défis à affronter au cours des prochaines années, en particulier dans un monde de publications électroniques, je crois que les changements actuellement en cours permettront à la $R C V$ de continuer à jouir d'une excellente santé pendant de nombreuses années à venir.

Les lecteurs sont invités à me faire part de leur opinion sur les changements décrits plus haut, ou sur tout autre aspect de la revue, en m'écrivant à l'adresse suivante: rosenber@post.queensu.ca.

Mark Rosenberg

Rédacteur en chef 